\section{References}

1. Arnold PG, Pairolero PC. Reconstruction of the radiation-damaged chest wall. Surg Clin North Am. 1989;69:1081-9.

2. Coleman JJ 3rd, Bostwick J. Rectus abdominis muscle-musculocutaneous flap in chest-wall reconstruction. Surg Clin North Am. 1989;69:1007-27.
3. Moon HK, Taylor GI. The vascular anatomy of rectus abdominis musculocutaneous flaps based on the deep superior epigastric system. Plast Reconstr Surg. 1988;82:815-32.

4. Fujiwara M, Nakamura Y, Sano A, Nakayama E, Nagasawa M, Shindo T. Delayed vertical rectus abdominis myocutaneous flap for anterior chest wall reconstruction. Aesthetic Plast Surg. 2006;30:120-4.

\title{
Combined bronchial sleeve resection and repair of partial anomalous pulmonary venous return
}

\author{
Robert L. Smith II, MD, George L. Zorn III, MD, Benjamin B. Peeler, MD, and David R. Jones, MD, Charlottesville, Va
}

$\mathrm{P}$ ulmonary sleeve resection for benign and malignant tumors of the proximal lobar bronchi preserves pulmonary function and avoids performance of a pneumonectomy. Partial anomalous pulmonary venous return (PAPVR) in an adult is a rare finding that is usually asymptomatic. We report a case of an obstructing left lower lobe carcinoid tumor combined with a PAPVR of the left superior pulmonary vein that was successfully managed without the need for pneumonectomy.

\section{Clinical Summary}

A 45-year-old female nonsmoker presented with a several-month history of fatigue, night sweats, fever, and moderate dyspnea. Multiple antibiotic treatments for left lower lobe pneumonia had failed. A chest roentgenogram demonstrated a left hilar mass and lower lobe collapse. Bronchoscopy demonstrated a $2 \times 2-\mathrm{cm}$ mass nearly occluding the distal left main stem bronchus and originating from the left lower lobe bronchus. Bronchial brushings and washings were nondiagnostic, but the mass was highly suspicious for a carcinoid tumor.

Computed tomography demonstrated a normal-appearing left upper lobe, confirmed the left lower lobe collapse, and identified a presumed anomalous left superior pulmonary vein draining into the left brachiocephalic vein (Figure 1). Pulmonary function studies showed a forced expiratory volume in 1 second of $54 \%$ of predicted value, a forced vital capacity of $55 \%$ of predicted value, and a ratio of $76 \%$ with a normal diffusion capacity. Quantitative pulmonary perfusion nuclear imaging revealed $4 \%$ perfusion to the left lung, with $96 \%$ to the right lung. Echocardiography failed to demonstrate any intracardiac shunts.

From the University of Virginia School of Medicine, Charlottesville, Va. Received for publication May 8, 2006; accepted for publication May 17, 2006.

Address for reprints: David R. Jones, MD, Department of Surgery, General Thoracic Surgery, PO Box 800679, University of Virginia, Charlottesville, VA 22908-0679 (E-mail: djones@ virginia.edu).

J Thorac Cardiovasc Surg 2006;132:982-3

0022-5223/\$32.00

Copyright $\odot 2006$ by The American Association for Thoracic Surgery doi:10.1016/j.jtcvs.2006.05.062

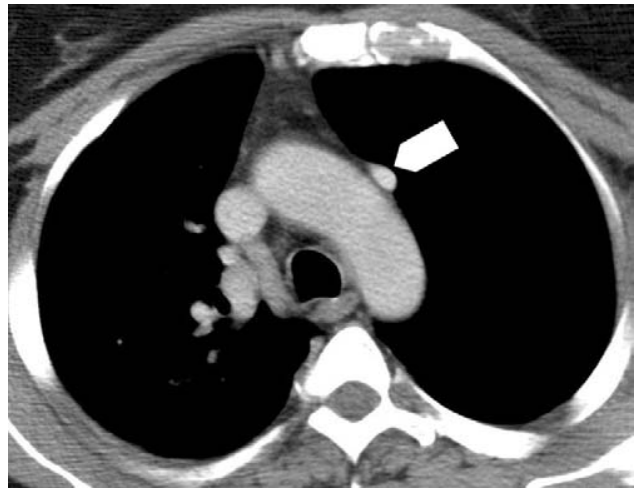

Figure 1. A preoperative computed tomogram identified an anomalous superior pulmonary vein (just lateral to the aortic arch and marked with an arrow) draining the left upper lobe and emptying into the left brachiocephalic vein.

Exploratory thoracotomy revealed a normal left upper lobe, a normal left inferior pulmonary vein, and a PAPVR of the left superior pulmonary vein into the left brachiocephalic vein. A left lower lobe sleeve resection was performed with mediastinal lymph node dissection. Frozen section analysis confirmed the diagnosis of a typical carcinoid tumor with histologically negative bronchial margins. The left superior pulmonary vein PAPVR was then managed by means of administration of systemic heparin, mobilization of the vein, and ligation at its insertion into the brachiocephalic vein. The pericardium was opened, and a vascular clamp was placed across the base of the left atrial appendage. The left superior pulmonary vein was then cut to an appropriate length and anastomosed to the atrial appendage (Figure 2).

After an uneventful recovery, the patient was discharged on postoperative day 4 . The final pathology revealed a T2 N0 M0 typical carcinoid tumor with postobstructive pneumonia of the lower lobe. A follow-up nuclear perfusion study at 3 months demonstrated enhanced flow to the left upper lobe. The patient has done well 30 months after the procedure, with follow-up bronchoscopy demonstrating a well-healed bronchial anastomosis. 


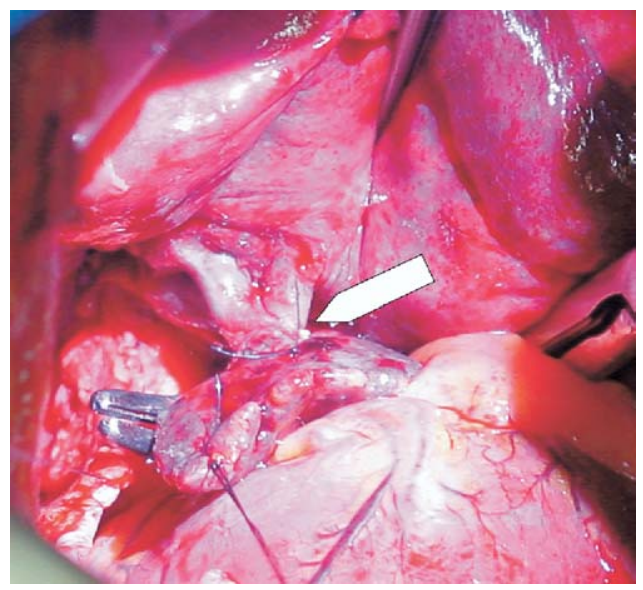

Figure 2. The left superior pulmonary vein was divided at its insertion into the left brachiocephalic vein, trimmed, and anastomosed to the left atrial appendage with a 5-0 Prolene suture. Shown here is the final anastomosis (marked with a white arrow), with the clamp remaining on the left atrial appendage.

\section{Discussion}

PAPVR is a relatively rare congenital anomaly, with a reported incidence of $0.2 \%$ to $0.7 \%$. ${ }^{1}$ Typically, these anomalies present during childhood, are right sided, are associated with atrial septal defects, and are twice as common in male patients. ${ }^{2}$ However, a recent review of chest computed tomograms in adults identified PAPVR anomalies more frequently in female patients, more commonly on the left, and infrequently associated with atrial septal defects. $^{3}$

There have been reports of pulmonary venous anatomic variants recognized at the time of thoracic surgical procedures. Khasati and colleagues ${ }^{4}$ report what was likely an anomalous left superior pulmonary vein that required anastomosis to the left atrial appendage at the time of lung transplantation. Sakurai and associates ${ }^{5}$ reported repair of a right PAPVR, followed by a left pneumonec- tomy 3 weeks later for lung cancer. These reports, combined with our case, suggest that thoracic surgeons need to consider concomitant pulmonary venous anomalies when planning pulmonary resections.

The surgical alternatives available for this patient included performing a left pneumonectomy or combining a bronchial sleeve resection with concomitant repair of the PAPVR. Although the patient would have tolerated a pneumonectomy based on her preoperative pulmonary assessment, we chose to spare the upper lobe and to hopefully improve her dyspnea by repairing her leftto-right shunt. The potential consequences of not correcting this anomaly could have been substantial. As evidenced by the preoperative pulmonary perfusion study, the bronchial obstruction and resultant pulmonary vasoconstriction severely limited the pulmonary blood flow to the left side and thus limited her left-to-right shunt. Failure to correct the PAPVR at the time of pulmonary resection might have potentially increased the shunt and led to increased right ventricular pressures.

To our knowledge, this is first reported case of an ipsilateral concomitant PAPVR repair and bronchial sleeve resection. This report highlights the fact that although PAPVR in an adult requiring pulmonary resection is uncommon, the mere presence of such a finding does not mandate performance of a pneumonectomy.

\section{References}

1. Alpert JS, Dexter L, Vieweg WVR, Haynes FW, Dalen JE. Anomalous pulmonary venous return with intact atrial septum: diagnosis and pathophysiology. Circulation. 1977;56:870-5.

2. Snellen HA, van Ingen HC, Hoefsmit EC. Patterns of anomalous pulmonary venous drainage. Circulation. 1968;38:45-63.

3. Haramati LB, Moche IE, Rivera VT, et al. Computed tomography of partial anomalous pulmonary venous connection in adults. J Comput Assist Tomogr. 2003;27:743-9.

4. Khasati NH, MacHaal A, Thekkudan S, Kumar S, Yonan N. An aberrant donor pulmonary vein during lung transplant: a surgical challenge. Ann Thorac Surg. 2005;79:330-1.

5. Sakurai H, Kondo H, Sekiguchi A, et al. Left pneumonectomy for lung cancer after correction of contralateral partial anomalous pulmonary venous return. Ann Thorac Surg. 2005;79:1778-80. 